\title{
Long Range Order and Atomic Connectivity in Two-Dimensional Square PbSe Nanocrystal Superlattices
}

Benjamin H. Savitzky ${ }^{1}$, Robert Hovden ${ }^{2}$, Kevin Whitham ${ }^{3}$, Tobias Hanrath ${ }^{4}$ and Lena F. Kourkoutis ${ }^{2,5}$

1. Department of Physics, Cornell University, Ithaca, NY 14853, USA.

2. School of Applied and Engineering Physics, Cornell University, Ithaca, NY 14853, USA.

3. Department of Materials Science and Engineering, Cornell University, Ithaca, NY 14853, USA.

4. School of Chemical and Biomolecular Engineering, Cornell University, Ithaca, NY 14853, USA.

5. Kavli Institute for Nanoscale Science, Cornell University, Ithaca, NY 14853, USA.

Two-dimensional materials have drawn significant attention due largely to their novel electronic band structures, yielding a wealth of potential applications in electronics and optoelectronics. Twodimensional materials based on superlattices (SLs) of semiconductor nanocrystals (NCs) have been recently demonstrated, and are particularly promising systems for band structure manipulation due to the number of independently tunable degrees of freedom, including nanocrystal size, shape, connectivity, and composition [1]. Inexpensive solution based synthesis make these systems viable candidates for scalable, efficient photovoltaics and other energy materials.

Long range order (LRO) as well as atomically coherent interfaces between NCs are key to bringing twodimensional NC SL materials to maturity [2]. Here we study a square SL of 6-7 nm PbSe NCs with grains of up to $3 \mu \mathrm{m}$, fabricated via self-assembly at a liquid-liquid interface. By combining atomicresolution aberration-corrected STEM data with large fields of view spanning entire NC SL grains (Fig. 1a) we have analyzed order at both length scales. Quantitative analysis of LRO was achieved using the pair correlation function $g(r)$, which describes the probability of finding two NC centers at a distance $r$ apart, normalized such that $g(r)=1$ represents no correlation between particle positions. For SLs $\sim 6 \mathrm{NC}$ layers thick, we find oscillations in $g(r)$ of $\pm 10 \%$ about unity out to $\sim 250 \mathrm{~nm}$, representing LRO in the sense that the NC positions remain statistically correlated out to distances of $250 \mathrm{~nm}$. For NC SL's only 2-3 layers thick $g(r)$ decays much faster, indicating faster loss of LRO (Fig. 1b). In contrast to a perfect crystal lattice, in which all lattice sites are specified exactly by the appropriate basis vectors, we find that these SLs behave as paracrystals, in which disorder between NCs is allowed to propagate through the lattice. We show that the structure of $g(r)$ closely matches a paracrystal model with an uncertainty in the inter-NC spacing of $\sigma=2.2 \AA$ for the 6 layer SL (Fig. 1c), while a paracrystal with $\sigma=3.5 \AA$ matches the 23 layer SL. In both cases, the decay in LRO appears to result from the propagation of atomic-scale disorder in the inter-NC spacing, and a greater disorder in NC-NC spacing causes faster loss of LRO in thinner SLs.

We analyzed single layer PbSe NC SLs both to understand the source of the short-range NC-NC positional disorder, and to push the system to its 2D limit. Monolayer SLs grew smaller grains, and displayed greater disorder in inter-NC spacing, consistent with the prior conclusion that thinner samples result in less robust crystallinity. Using statistical analysis we further show that the presence or absence of a continuous atomic lattice connecting two adjacent NCs directly impacts the NC-NC spacing, with unconnected adjacent NCs $8.0 \AA$ farther away from each other on average than connected NCs (Fig. 2). Interestingly, the disorder in inter-NC spacing for adjacent, connected NCs in the monolayer (3.9Å) was similar to that in the $\sim 2-3$ layer SL $(3.5 \AA)$. However, the overall disorder in inter-NC spacing for the monolayer SL is significantly larger $(5.3 \AA)$ due to the presence of unconnected NCs. Our data suggest that the loss of large grains and LRO in monolayer SLs is a result of reduced NC-NC connectivity. 
[1] T. Mokari, M. Zhang, and P. Yang, J. Am. Chem. Soc. 129 (2007), 9864-9865

[2] M.P. Boneschanscher et al., Science 344 (2014), 1377-1380

[3] BHS acknowledges support from the NSF IGERT (DGE-0903653). This work was supported by the Cornell Center for Materials Research with funding from the NSF MRSEC program (DMR-1120296)
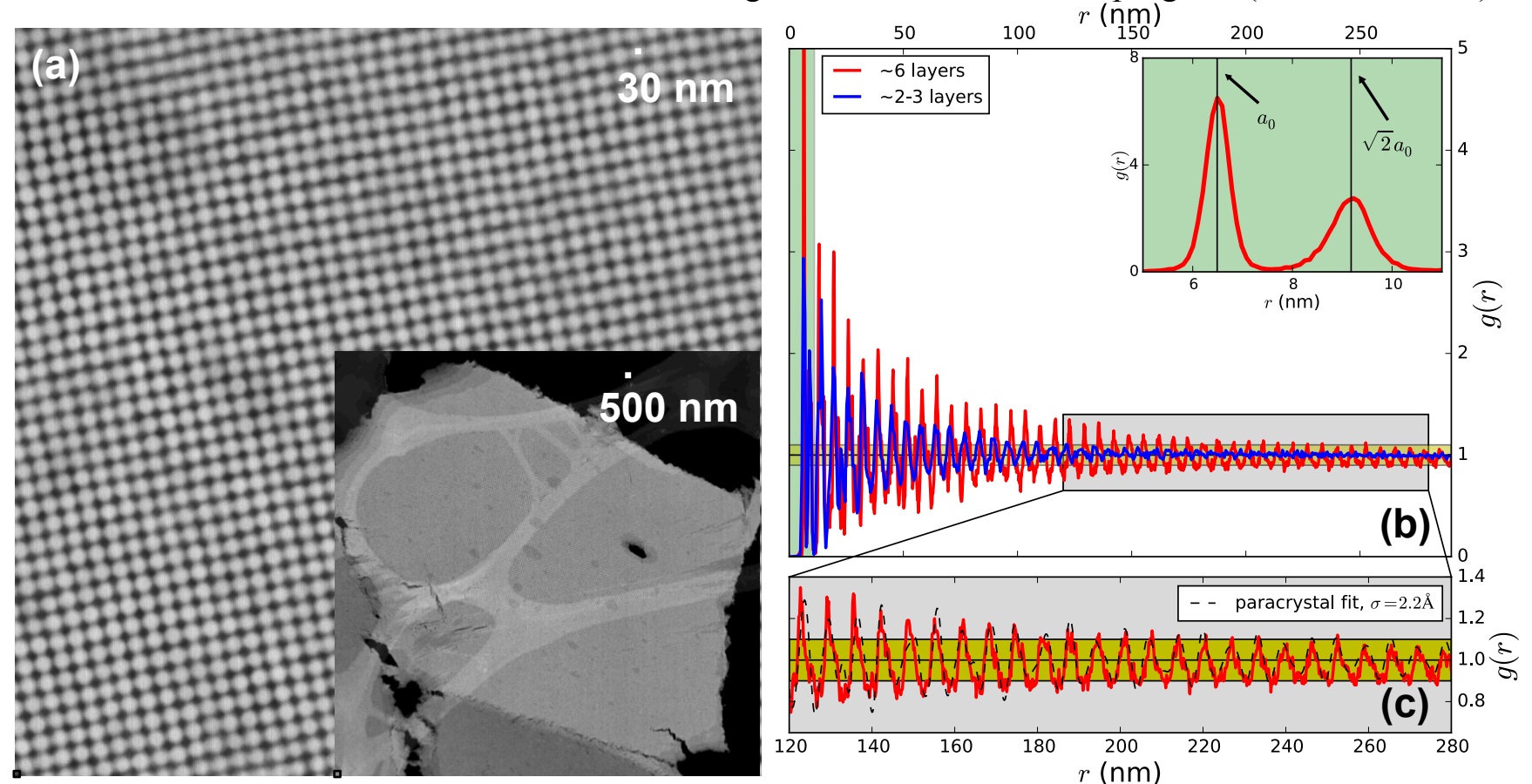

Figure 1. Long range order (LRO) in PbSe nanocrystal (NC) superlattices (SLs) $(a)$ is analyzed using the pair correlation function, $g(r)$. Good LRO in SLs $\sim 6 \mathrm{NC}$ layers thick is evident from continued oscillations of $\pm 10 \%$ about unity (yellow bar) at distances of $\sim 250 \mathrm{~nm}$, while SLs $\sim 2-3$ NC layers thick have decreased LRO, shown by the faster decay of $g(r)(b) . g(r)$ closely matches a paracrystalline model, indicating that unlike in perfect crystals, NC positional disorder propagates through the SL $(c)$.
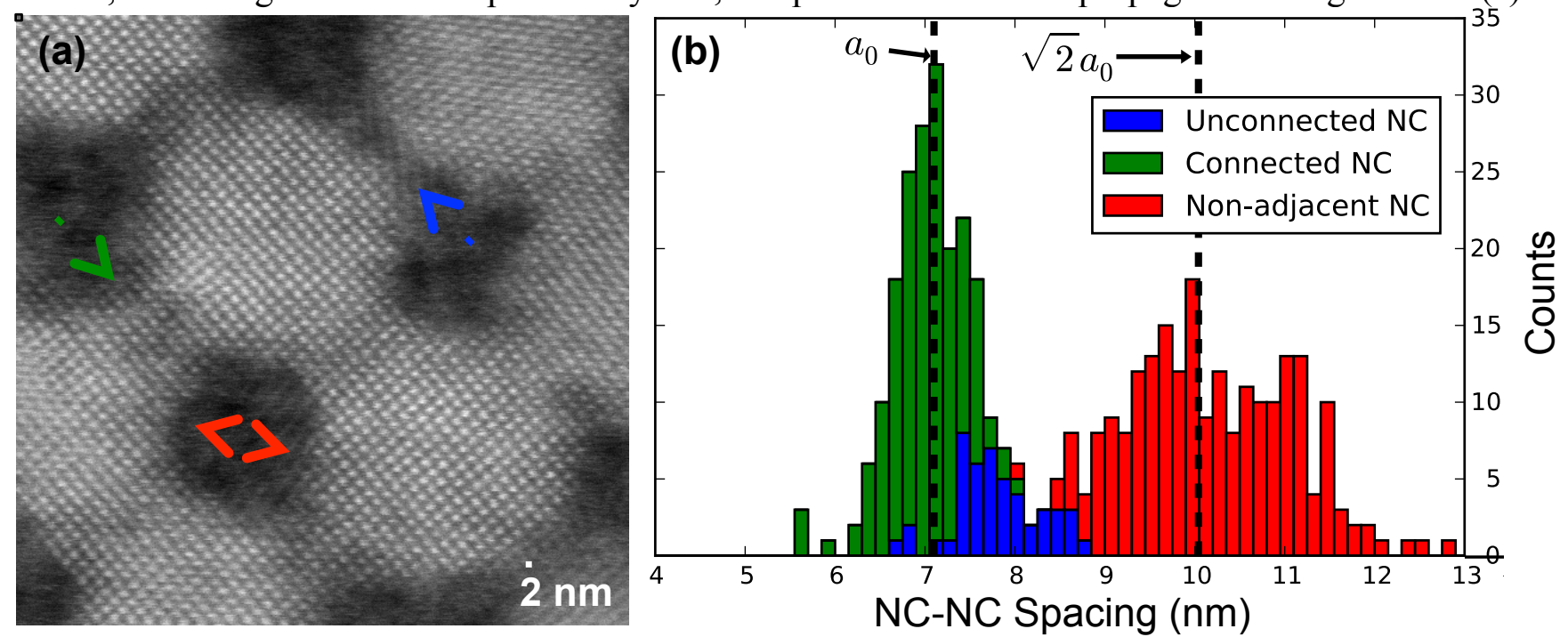

Figure 2. Statistical analysis of atomic-scale connectivity in a one-layer thick PbSe nanocrystal superlattice. Disorder in inter-NC spacing in monolayers is due in part to variation in the NC-NC connectivity (a). Adjacent, connected NCs (green) have a mean spacing of $7.0 \pm 0.39 \mathrm{~nm}$, while adjacent, unconnected NCs (blue) have an increased mean spacing of $7.8 \pm 0.48 \mathrm{~nm}(b)$. 